\title{
Pathological findings following radical prostatectomy in patients who are candidates for active surveillance: impact of varying PSA levels
}

\author{
Dong Il Kang ${ }^{1,2}$, Thomas L. Jang ${ }^{1}$, Jeongyun Jeong ${ }^{1}$, Eun Young Choi ${ }^{1}$, Kelly Johnson ${ }^{1}$, Dong Hyeon Lee ${ }^{3}$, \\ Wun-Jae Kim ${ }^{4}$ and Isaac Yi Kim ${ }^{1}$
}

Active surveillance is an acceptable treatment option in men with a low-risk prostate cancer. In the present study, we have retrospectively reviewed the outcomes of 509 men who fit the criteria for active surveillance but selected radical prostatectomy. Then, the impact of varying prostate-specific antigen (PSA) levels on the risk of upstaging and upgrading in these patients was assessed. Pathological characteristics of patients who fulfilled the inclusion criteria under three active surveillance criteria-those of the University of California-San Francisco, the National Cancer Institute and the European Association of Urology-were examined. The proportion of men who were deemed candidates for active surveillance but were subsequently upstaged or upgraded was determined. Of 509 patients, $186(36.5 \%), 132(25.9 \%)$ and $88(17.3 \%)$ men fulfilled the active surveillance criteria, respectively. Upgrading (Gleason scores $7-10$ ) ranged from $32.8 \%$ to $38.6 \%$, while upstaging ( $\geqslant \mathrm{pT} 3$ ) ranged from $10.2 \%$ to $12.5 \%$, depending on the three active surveillance criteria. After a median follow-up of 24 months, three patients developed a biochemical recurrence. When the impact of varying PSA levels was examined using a test for trend analysis in the context of PSA for each protocol, rates of upstaging were lower in men with PSA $<\mathbf{4} \mathrm{n} \mathrm{ml}^{-1}$. However, there was no impact of varying PSA levels on upgrading. In conclusion, commonly used active surveillance protocols carry the risks of upgrading and upstaging. More reliable and accurate markers are needed to better stratify the risks of men who are appropriate candidates for active surveillance.

Asian Journal of Andrology (2011) 13, 838-841; doi:10.1038/aja.2011.54; published online 25 July 2011

Keywords: prostatectomy; prostate-specific antigen; prostatic neoplasm; risk

\section{INTRODUCTION}

Over $90 \%$ of newly diagnosed prostate cancer (PCa) cases in the United States are clinically localized, and the 5-year survival rates in these men are nearly $100 \% .{ }^{1}$ The introduction of prostate-specific antigen (PSA)-based screening has led to the increased detection of potentially more indolent tumours with low risk. Consequently, a subgroup of these men may be subjected to unnecessary treatment with associated morbidities. As such, active surveillance (AS) has emerged as a viable treatment option for selected patients with lowrisk $\mathrm{PCa}{ }^{2}$

AS criteria that accurately predict which individuals have truly indolent disease while simultaneously providing guidelines for serially monitoring and rapidly identifying patients with disease progression have been debated. ${ }^{3}$ Ideally, the strictest criteria should be used to avoid misclassifying men with non-indolent tumours as eligible for AS. Despite these efforts, high-risk PCas have been reported in men who are candidates for AS, as commonly used AS guidelines do not always accurately predict pathological outcomes. ${ }^{3-5}$

The aim of our study was to examine the outcomes in men who fit the criteria for AS but selected radical prostatectomy and to investigate the impact of varying PSA threshold. The impact of varying PSA threshold was used in an attempt to further refine the AS protocols.

\section{MATERIALS AND METHODS}

Patients

After obtaining Institutional Review Board approval of Cancer Institute of New Jersey, the data of 509 patients who had at least 12 core biopsies and selected robot-assisted radical prostatectomy (RARP) using the DaVinci system (Intuitive Surgical, Sunnyvale, CA, USA) between January 2004 and October 2009 were reviewed. All men in this group had a life expectancy greater than 10 years. Of the 509 men, 406 patients with clinical and pathological data that met the most stringent AS guidelines were reviewed.

Three guidelines were analysed in this study: University of California-San Francisco (UCSF)-PSA $<10 \mathrm{ng} \mathrm{ml}^{-1}$, biopsy Gleason sum $\leqslant 6$ with no pattern 4 or 5 , cancer involvement of $<33 \%$ of biopsy cores, clinical stage T1/T2a tumour; ${ }^{6}$ National Cancer Institute (NCI) - (i) life expectancy $<10$ years with PSA $<10 \mathrm{ng} \mathrm{ml}^{-1}$, clinical stage $\leqslant \mathrm{T} 2 \mathrm{a}$, Gleason sum $\leqslant 6$; or (ii) life expectancy up to 20 years with PSA $<10 \mathrm{ng} \mathrm{ml}^{-1}$, clinical stage

${ }^{1}$ Section of Urologic Oncology and Dean and Betty Gallo Prostate Cancer, The Cancer Institute of New Jersey, Robert Wood Johnson Medical School, New Brunswick, NJ 08903, USA; ${ }^{2}$ Department of Urology, Inje University, Medical School, Busan 614-735, Korea; ${ }^{3}$ Department of Urology, Ewha Womans University, Seoul 158-710, Korea and ${ }^{4}$ Department of Urology, Chungbuk National University College of Medicine, Cheongju 361-711, Korea 
$\leqslant \mathrm{T} 2 \mathrm{a}$, Gleason score $\leqslant 6$, fewer than three biopsy cores, $\leqslant 50 \%$ positive core (http://www.cancer.gov/aboutnci/ncicancerbulletin/archive/ 2010/011210/page2); European Association of Urology (EAU)-(i) life expectancy $<10$ years with PSA $<10 \mathrm{ng} \mathrm{ml}^{-1}$, biopsy Gleason score $\leqslant 6$ and clinical stage cT1c-cT2a; or (ii) men with a life expectancy $>15$ years with PSA $<10 \mathrm{ng} \mathrm{ml}^{-1}$, biopsy Gleason score $\leqslant 6$, cT1ccT2a, fewer than three biopsy cores, and maximum positive core $<50 \%$. $^{2}$ Life expectancy for males in the US is 75.1 years. ${ }^{7}$

Following surgery, pathological stage and Gleason score were examined. Patients with follow-up $<3$ months were excluded. Biochemical recurrence was defined as three consecutive rises in PSA $>0.2 \mathrm{ng} \mathrm{ml}^{-1}$. The following definitions were used: upstaging - from T1-T2 to T3-T4; upgrading-pathological Gleason score $\geqslant 7$. Based on the analysis of the three AS guidelines, we examined the effect of lowering the PSA threshold to 8,6 and $4 \mathrm{ng} \mathrm{ml}^{-1}$ on pathological characteristics. Our institute criteria (Cancer Institute of New Jersey (CINJ)'s criteria) for AS are PSA $<4 \mathrm{ng} \mathrm{ml}^{-1}$, biopsy Gleason sum $\leqslant 6$ with no pattern 4 or 5, and clinical stage T1/T2a tumour, and 509 RARP cases were analysed.

\section{Statistical analysis}

Statistical analysis was done by using SPSS ver. 18.0 (SPSS Inc., Chicago, IL, USA). The results were analysed statistically using ANOVA, Chi-square test and test for trends (linear-by-linear association). Kaplan-Meier curve with log-rank test was used to analyse the biochemical recurrence rates. $P$ values $<0.05$ were considered significant.

\section{RESULTS}

Of 509 PCa patients who underwent RARP, 186 (36.5\%), 132 (25.9\%), $88(17.3 \%)$ and $75(14.7 \%)$ men fulfilled the AS criteria of UCSF, NCI, EAU and CINJ, respectively. None of these patients with low-risk PCa received additional preoperative CT, MRI or bone scan. As shown in Table 1, there were statistically significant differences among the four groups in age, preoperative PSA and clinical T stage. Overall, about $90 \%$ of the patients had clinical stage T1c disease.

The percentage of patients who were upgraded (Gleason scores $\geqslant 7$ ) ranged from $32.8 \%$ to $38.6 \%$, while those who were upstaged ( $\geqslant$ pT3) ranged from $10.2 \%$ to $12.5 \%$ (Table 2). High-grade PCas or specimens with a primary Gleason grade $\geqslant 4$ were found in $8.6 \%$ to $13.6 \%$ of patients (Table 2). After a median follow-up of 24 months (range: 3-48 months), two patients (patients 1 and 2) who fit the NCI's and EAU's AS criteria developed a PSA recurrence, respectively. The two patients and another one exhibited biochemical evidence of recurrence using the UCSF guidelines (Table 2). The specific details of the three patients are shown in Table 3. After modifying the PSA criteria for the AS protocols to $<10,<8,<6$ and $<4$, upstaging rates decreased (test for trends, $P<0.05$ ) (Figure 1 and Table 4). Kaplan-Meier curves demonstrated no differences in PSA biochemical recurrence-free survival among four AS protocols (three external AS protocols and our institutional AS with PSA $<4 \mathrm{ng} \mathrm{ml}^{-1}, P=0.619$ ) (Figure 2).

\section{DISCUSSION}

In the present study, we analysed three commonly available AS guidelines in the context of post-RARP pathological outcomes. Of 509 patients, 186, 132 and 88 patients met UCSF, NCI, and EAU criteria, respectively. There were no significant differences in the preoperative parameters among three AS protocols except for age because NCI and EAU guidelines specify life expectancy as part of the inclusion criteria.

With the implementation of population-based screening for $\mathrm{PCa}$, many experts have expressed concern for the overtreatment of PCa. Indeed, it is estimated that up to $\sim 80 \%$ of all treated PCa cases are unnecessary. ${ }^{8,9}$ Overtreatment of PCa adds not only to the rising healthcare costs but also to the long-term decline in quality of life due to factors such as incontinence and impotence. However, at our institution, the long-term postoperative continence and potency rates are over $90 \%,{ }^{10}$ while the major complication rate (Clavien class III or higher) was $3 \%,{ }^{11}$ suggesting that the impact of robotic surgical intervention on quality of life for PCa is mostly short lived for our patients. Regardless, as a tool to decrease the incidence of prostate cancer overtreatment, active surveillance has gained significant support over the last decade. That said that there is still a lack of uniform standards and criteria for active surveillance in men with low-risk PCa. ${ }^{9}$

Our data showed that over one-third of patients who met AS protocols were upgraded after radical prostatectomy (Gleason scores 7-10). Although Gleason scores 6 and 7 are both considered intermediate risk, patients with Gleason sum 7 have higher rates of biochemical recurrence and distant metastasis. Interestingly, about $30 \%$ of patients who were upgraded have Gleason score $4+3$ patterns. Multiple investigators have suggested that Gleason score $4+3$ tumours are more aggressive than Gleason sum $3+4$ tumours. ${ }^{12,13}$ For example, Stark et al. ${ }^{12}$ reported that Gleason score $4+3$ tumours, when compared with Gleason score $3+4$ tumours, were associated with a threefold higher incidence of bone metastasis and prostate cancer-related death. Therefore, published AS guidelines should consider the differential outcomes of $3+4$ versus

Table 1 Characteristics of patients enrolled in four active surveillances. Data were expressed as median (range)

\begin{tabular}{|c|c|c|c|c|c|c|}
\hline & UCSF's Criteria & NCl's Criteria & EAU's criteria & \multirow{2}{*}{ P value* } & CINJ's criteria & \multirow{2}{*}{$\mathrm{P}$ value ${ }^{* *}$} \\
\hline & 186 & 132 & 88 & & 75 & \\
\hline Age (year) & $60.0(36-77)$ & $62.0(55-77)$ & $64.0(60-77)$ & $0.0001^{a}$ & $57.0(36-77)$ & $0.0001^{a}$ \\
\hline $\mathrm{BMI}\left(\mathrm{kg} \mathrm{m}^{-2}\right)$ & $28.6(19.9-42.0)$ & $28.4(20.2-42.0)$ & $28.2(20.2-39.7)$ & $0.286^{a}$ & $28.2(21.5-41.9)$ & $0.388^{a}$ \\
\hline $\begin{array}{l}\text { Preoperative Serum PSA } \\
\left(\mathrm{ng} \mathrm{ml^{-1 } )}\right.\end{array}$ & $4.5(0.4-9.8)$ & $4.7(0.6-9.8)$ & $4.9(0.9-9.8)$ & $0.075^{a}$ & $3.2(0.4-3.9)$ & $0.0001^{a}$ \\
\hline Biopsy Gleason score & 6 & 6 & 6 & $1.000^{\mathrm{a}}$ & 6 & $1.000^{\mathrm{a}}$ \\
\hline Clinical stage, \% (n) & & & & $0.774^{\mathrm{b}}$ & & $0.018^{\mathrm{b}}$ \\
\hline $\mathrm{T} 1$ & $89.8(167)$ & $91.7(121)$ & $92.0(81)$ & & 78.7 (59) & \\
\hline $\mathrm{T} 2 \mathrm{a}$ & $10.2(19)$ & $8.3(11)$ & $8.0(7)$ & & $21.3(16)$ & \\
\hline
\end{tabular}

Abbreviations: BMI, body mass index; CINJ, Cancer Institute of New Jersey; NCI, National Cancer Institute; PSA, prostate-specific antigen; UCSF, University of CaliforniaSan Francisco.

a ANOVA.

${ }^{\mathrm{b}} \chi^{2}$ test.

* Pvalue: UCSF vs. NCI vs. EAU.

**P value: UCSF vs. $\mathrm{NCI}$ vs. EAU vs. CINJ. 
Table 2 Changes in upstaging, upgrading and PSA biochemical recurrence according to active surveillance criteria

\begin{tabular}{lccc}
\hline & UCSF's criteria & NCl's criteria & EAU's criteria \\
\cline { 2 - 3 } Upstaging, $n(\%)$ & $(n=186)$ & $(n=132)$ & $(n=88)$ \\
T0 & $19(10.2)$ & $14(10.6)$ & $11(12.5)$ \\
T2 & $2(1.1)$ & $1(0.8)$ & $1(1.1)$ \\
T3a & $165(88.7)$ & $117(88.6)$ & $76(86.4)$ \\
Upgrading, $n(\%)$ & $19(10.2)$ & $14(10.6)$ & $11(12.5)$ \\
$\leqslant 6$ & $61(32.8)$ & $47(35.6)$ & $34(38.6)$ \\
$7(3+4)$ & $125(67.2)$ & $85(64.4)$ & $54(61.4)$ \\
$7(4+3)$ & $45(24.2)$ & $31(23.5)$ & $22(25.0)$ \\
$\geqslant 8$ & $10(5.4)$ & $9(6.8)$ & $6(6.8)$ \\
PSA recurrence, $n(\%)$ & $6(3.2)$ & $7(5.3)$ & $6(6.8)$ \\
\hline
\end{tabular}

Abbreviations: EAU, European Association of Urology; NCI, National Cancer Institute; PSA, prostate-specific antigen; UCSF, University of California-San Francisco.

$4+3$ cancers. In the context of upstaging, $10.2 \%-12.5 \%$ of men who fulfilled the criteria for AS showed a pathological stage of pT3a or higher. This is consistent with studies that have reported that $5 \%-26 \%$ of lowrisk patients are upstaged following surgery. ${ }^{14-17}$ These relatively high percentages of upgrading and upstaging suggest that the AS protocols need further refinement to increase accuracy.

In an effort to improve the recommended AS guidelines, we investigated the effect of varying PSA levels on the rate of upstaging and upgrading. Previously, it has been reported that the proportion of men on AS protocols whose tumours were upstaged ranged from $7 \%-19 \%$ in men with PSA $<10 \mathrm{ng} \mathrm{ml}^{-1}, 14 \%-24.8 \%$ in men with PSA $<15 \mathrm{ng} \mathrm{ml}^{-1}$ and $18 \%-26 \%$ in men with PSA $<20 \mathrm{ng}$ $\mathrm{ml}^{-1}$. 3,4,15,17 Moreover, modifying the D'Amico criteria for low-risk prostate cancer to PSA $\leqslant 4 \mathrm{ng} \mathrm{ml}^{-1}$ resulted in reduced rates of extracapsular extension, from $13.5 \%$ to $6.9 \%$. $^{4,18}$

Based on these findings, the CINJ has adopted the following criteria for AS: PSA $<4 \mathrm{ng} \mathrm{ml}^{-1}$, biopsy Gleason sum $<7$ and clinical stage $\leqslant \mathrm{T} 2 \mathrm{a}$; life expectancy is currently not a part of our institutional guideline. This modified CINJ protocol for AS needs to be confirmed in a large-scale study.

Consistent with these observations, we found through trend analysis that the percentages of patients who were upstaged decreased with lower PSA threshold $(P<0.05)$. When PSA $<4 \mathrm{ng} \mathrm{ml}^{-1}$ was used, the number of patients who were upstaged under the three AS criteria decreased. However, because our cohort data are limited, we could not perform a multivariate analysis. Notwithstanding, the use of a

Table 3 Characteristics of patients with biochemical recurrence following radical prostatectomy

\begin{tabular}{|c|c|c|c|}
\hline & Patient 1 & Patient 2 & Patient 3 \\
\hline \multicolumn{4}{|l|}{ Preoperative } \\
\hline Age (years) & 60 & 66 & 42 \\
\hline $\mathrm{BMI}\left(\mathrm{kg} \mathrm{m}^{-2}\right)$ & 24.5 & 29.0 & 33.7 \\
\hline Pre-PSA level (ng ml $\left.{ }^{-1}\right)$ & 5.8 & 9.8 & 8.5 \\
\hline Biopsy Gleason score & $3+3$ & $3+3$ & $3+3$ \\
\hline Positive core & 2 & 3 & 1 \\
\hline Maximum core $(\%)$ & 15 & 35 & 20 \\
\hline Clinical T stage & $\mathrm{T} 1 \mathrm{c}$ & $\mathrm{T} 1 \mathrm{c}$ & $\mathrm{T} 2 \mathrm{a}$ \\
\hline \multicolumn{4}{|l|}{ Postoperative } \\
\hline Pathological T stage & $\mathrm{T} 2 \mathrm{c}$ & ТЗа & ТЗа \\
\hline Pathological Gleason score & $3+3$ & $4+3$ & $3+4$ \\
\hline Positive surgical margin & apex & $(-)$ & $(-)$ \\
\hline $\begin{array}{l}\text { Time to PSA biochemical } \\
\text { recurrence (months) }\end{array}$ & 3 & 24 & 48 \\
\hline
\end{tabular}

Abbreviations: BMI, body mass index; PSA, prostate-specific antigen. lower PSA cutoff did not help decrease the rate of upgrading and biochemical recurrence in those who underwent radical prostatectomy. Recently, it has been reported that PSA kinetics is not reliable in predicting disease progression in the context of active surveillance for prostate cancer. ${ }^{19}$ Together with the results of the present work, it is likely that the PSA criteria for active surveillance and indications for intervention require further refinement.

It should also be noted that by decreasing the PSA threshold, the number of patients who are candidates for active surveillance will decrease. In the present study, the use of PSA $<4 \mathrm{ng} \mathrm{ml}^{-1}$ decreased the number of patients who are eligible for AS by nearly $75 \%$. Reduction in the number of eligible patients by using with a lower PSA cutoff will increase the number of overtreated patients. Thus, as a whole, the gain in accuracy achieved by lowering the PSA threshold for active surveillance may not offset the potential cost of overtreatment and resulting complications. This hypothesis will be investigated in the future using a larger cohort of patients.

The present study has several limitations. First, the median follow-up of 24 months is relatively short. Thus, additional time is needed to
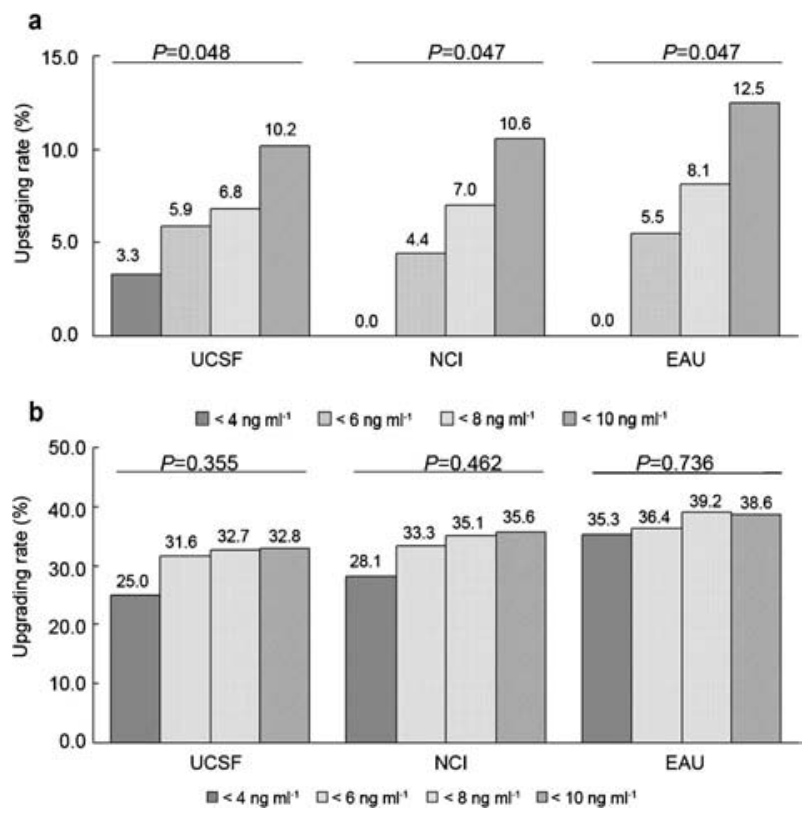

Figure 1 Changes in upstaging (a) and upgrading (b) according to PSA level. $P$ value: linear-by-linear association. EAU, European Association of Urology; $\mathrm{NCl}$, National Cancer Institute; UCSF, University of California-San Francisco. 
Table 4 Results of modified active surveillance criteria with PSA $<4 \mathrm{ng} \mathrm{ml}^{-1}$

\begin{tabular}{|c|c|c|c|c|c|}
\hline & Modified UCSF & Modified $\mathrm{NCl}$ & Modified EAU & CINJ criteria & \\
\hline & $(n=60)$ & $(n=32)$ & $(n=17)$ & $(n=75)$ & \\
\hline Upstaging, n (\%) & $2(3.3)$ & $0(0)$ & $0(0)$ & $3(4.0)$ & 0.589 \\
\hline Upgrading, n (\%) & $15(25.0)$ & $9(28.2)$ & $6(35.3)$ & $23(30.6)$ & 0.901 \\
\hline$\leqslant 6$ & $45(75.0)$ & 23 (71.9) & $11(64.7)$ & $52(68.0)$ & \\
\hline $7(3+4)$ & $12(20.0)$ & $6(18.8)$ & $3(17.6)$ & $19(25.3)$ & \\
\hline $7(4+3)$ & $2(3.3)$ & $2(6.3)$ & $2(11.8)$ & $3(4.0)$ & \\
\hline$\geqslant 8$ & $1(1.7)$ & $1(3.1)$ & $1(5.9)$ & $1(1.3)$ & \\
\hline PSA recurrence, $n(\%)$ & $0(0.0)$ & $0(0.0)$ & $0(0.0)$ & $0(0.0)$ & 1.000 \\
\hline
\end{tabular}

Abbreviations: CINJ, Cancer Institute of New Jersey; EAU, European Association of Urology; NCI, National Cancer Institute; PSA, prostate-specific antigen; UCSF, University of California-San Francisco.

determine the association between the impact on upgrading/upstaging and the biochemical recurrence rate. Indeed, neoadjuvant androgen ablation studies have clearly demonstrated that changes in pathological findings may not predict clinical outcome in men undergoing radical prostatectomy. Second, most patients in this study underwent prostate biopsies at outside institutions. To minimize the impact of this variable, all biopsy results were reviewed by one pathologist at our institution and only those with 12 or more core biopsies were included in this study. Finally, the cohort itself is small. As a result, more rigorous statistical studies including multivariate analysis could not be performed.

In conclusion, even the most current and stringent criteria for AS have a significant risk of upgrading and upstaging. More reliable and accurate markers are needed to better stratify the risks of men who are appropriate candidates for AS. To this end, results of the present study suggest that decreasing the PSA cutoff to $4 \mathrm{ng} \mathrm{ml}^{-1}$ did not help significantly decrease the risk of recurrence in men who opted for active surveillance. Additional studies using a larger cohort of patients with a longer period of follow-up are necessary to confirm our findings.

\section{AUTHOR CONTRIBUTIONS}

DIK designed this study, drafted the manuscript and performed the statistical analysis. TLJ participated in its design and helped to draft the manuscript. JJ participated in its design, interpretation of data and performed the statistical analysis. EYC participated in its design and interpretation of data. KJ participated in acquisition and interpretation of data. DHL and WK participated in interpretation of data and helped to draft the manuscript. IYK designed this study and drafted the manuscript.

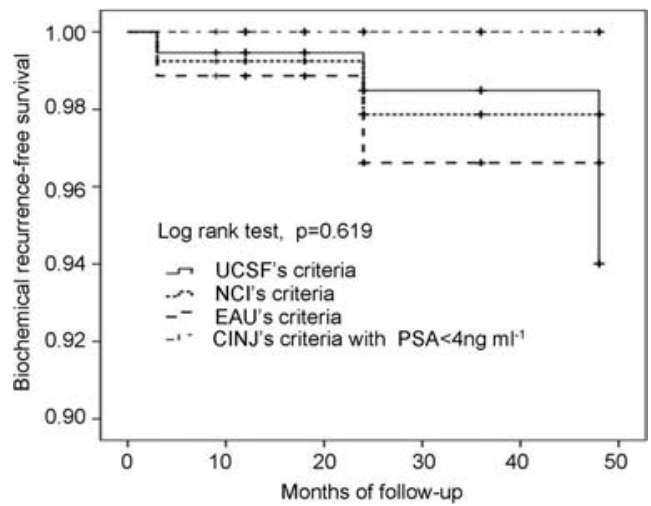

Figure 2 PSA biochemical recurrence-free survival following radical prostatectomy. CINJ, Cancer Institute of New Jersey.; EAU, European Association of Urology; NCI, National Cancer Institute; PSA, prostate-specific antigen; UCSF, University of California-San Francisco.

\section{COMPETING FINANCIAL INTERESTS}

The authors declare no competing financial interests

\section{ACKNOWLEDGMENTS}

This work was supported by the Inje Research and Scholarship Foundation in 2009, Korea.

1 Jemal A, Siegel R, Ward E, Hao Y, Xu J et al. Cancer statistics, 2008. CA Cancer J Clin 2008; 58: 71-96.

2 Heidenreich A, Aus G, Bolla M, Joniau S, Matveev VB et al. EAU guidelines on prostate cancer. Eur Urol 2008; 53: 68-80.

3 Thaxton CS, Loeb S, Roehl KA, Kan D, Catalona WJ. Treatment outcomes of radical prostatectomy in potential candidates for 3 published active surveillance protocols. Urology 2010; 75: 414-8.

4 Suardi N, Capitanio U, Chun FK, Graefen M, Perrotte P et al. Currently used criteria for active surveillance in men with low-risk prostate cancer: an analysis of pathologic features. Cancer 2008; 113: 2068-72.

5 Kattan MW, Eastham JA, Wheeler TM, Maru N, Scardino PT et al. Counseling men with prostate cancer: a nomogram for predicting the presence of small, moderately differentiated, confined tumors. J Urol 2003; 170: 1792-7.

6 Dall'Era MA, Konety BR, Cowan JE, Shinohara K, Stauf F et al. Active surveillance for the management of prostate cancer in a contemporary cohort. Cancer 2008; 112: 2664-70.

7 Heron M, Hoyert DL, Murphy SL, Xu J, Kochanek KD et al. Deaths: final data for 2006. Natl Vital Stat Rep 2009; 57: 1-134.

8 McGregor M, Hanley JA, Boivin JF, McLean RG. Screening for prostate cancer: estimating the magnitude of overdetection. CMAJ 1998; 159: 1368-72.

9 Harnden P, Naylor B, Shelley MD, Clements H, Coles B et al. The clinical management of patients with a small volume of prostatic cancer on biopsy: what are the risks of progression? A systematic review and meta-analysis. Cancer 2008; 112: 971-81.

10 Potdevin L, Ercolani M, Jeong J, Kim IY. Functional and oncologic outcomes comparing interfascial and intrafascial nerve sparing in robot-assisted laparoscopic radical prostatectomies. J Endourol 2009; 23: 1479-84.

11 Jeong J, Choi EY, Kim IY. Clavien classification of complications after the initial series of robot-assisted radical prostatectomy: the Cancer Institute of New Jersey/Robert Wood Johnson Medical School experience. J Endourol 2010; 24: 1457-61.

12 Stark JR, Perner S, Stampfer MJ, Sinnott JA, Finn S et al. Gleason score and lethal prostate cancer: does $3+4=4+3$ ? J Clin Oncol 2009; 27: 3459-64.

13 Chan TY, Partin AW, Walsh PC, Epsein JI. Prognostic significance of Gleason score $3+4$ versus Gleason score $4+3$ tumor at radical prostatectomy. Urology 2000; 56: 823-7.

14 Louie-Johnsun M, Neill M, Treurnicht K, Jarmulowicz M, Eden C. Final outcomes of patients with low-risk prostate cancer suitable for active surveillance but treated surgically. BJU Int 2009; 104: 1501-4.

15 Ploussard G, Salomon L, Xylinas E, Allory Y, Vordos D et al. Pathological findings and prostate specific antigen outcomes after radical prostatectomy in men eligible for active surveillance-does the risk of misclassification vary according to biopsy criteria? J Urol 2010; 183: 539-44.

16 Richstone L, Bianco FJ, Shah HH, Kattan MW, Eastham JA et al. Radical prostatectomy in men aged $>$ or $=70$ years: effect of age on upgrading, upstaging, and the accuracy of a preoperative nomogram. BJU Int 2008; 101: 541-6.

17 Conti SL, Dall'era M, Fradet V, Cowan JE, Simko J et al. Pathological outcomes of candidates for active surveillance of prostate cancer. J Urol 2009; 181: 1628-33.

18 DAmico AV, Coleman CN. Role of interstitial radiotherapy in the management of clinically organ-confined prostate cancer: the jury is still out. J Clin Oncol 1996; 14: 304-15.

19 Ross AE, Loeb S, Landis P, Partin AW, Epstein Jl et al. Prostate-specific antigen kinetics during follow-up are an unreliable trigger for intervention in a prostate cancer surveillance program. J Clin Oncol 2010; 28: 2810-6. 